\title{
CONTINUOUS BARRIER SEPARATION OF WEAKLY MAGNETIC ORES IN THE FIELD OF REPULSIVE MAGNETIC FORCE
}

\author{
A.M. TURKENICH, E.S. LAPSHIN AND V.I. DUDNIK \\ Institute of Geotechnical Mechanics of the Academy of Sciences \\ of the Ukraine, 320005 Dnepropetrovsk, Ukraine
}

(Received March 19, 1996, accepted June 5, 1996)

\begin{abstract}
$\underline{\text { Abstract }}$
A new method of continuous HIMS with repulsive magnetic force has been worked out. It creates a separator with an output similar to that of rotary separators and with higher recovery of the magnetic fraction. The separators operates without any moving parts. Results of investigation of this method are summarised.
\end{abstract}

Rotary magnetic separators have been spread widely for beneficiation of fine weakly magnetic ores with a wide range of magnetic susceptibility of mineral particles. They are based on adhesion of magnetic particles to ferromagnetic bodies (matrix) under the action of attractive magnetic force. Non-magnetic particles pass through the gaps between the ferromagnetic bodies and report into the tailings.

Because the magnetic particles must be washed off from the ferromagnetic bodies, the rotor moves these bodies out of the magnetic field to a position of water rinse. Such a rotation requires up to $25 \%$ of total energy consumption and the rotor has the size and the cost which are equal to one half of the total mass and price of the separator. These numbers are considerable. For instance, a separator 6ERM-35/315 weighs 150 tonnes, has the energy consumption of up to $140 \mathrm{kWh}$ and occupies $30 \mathrm{~m}^{2}$ of floor space. 
However, a beneficiation of a variety of ores with narrow range of magnetic susceptibility of particles does not require such a large expenditure, if instead of attractive magnetic force, a repulsive magnetic force could be used. Such a technique of magnetic separation was developed by Magnetic and Hydraulics Technologies Co. and a static separator has been built. A material to be separated is fed into the matrix and magnetic and non-magnetic fractions are discharged from the matrix continuously. The separator does not have any moving parts. Absence of a rotor allows to achieve reduction in the mass of the separator and its energy consumption.

This method of continuous separation is based on creation of magnetic barrier with the help of the repulsive magnetic force in the path of the movement of material. The non-magnetic particles pass freely through the magnetic barrier and report into the tailings. The magnetic particles cannot overcome the magnetic barrier and move along the barrier into the magnetic product.

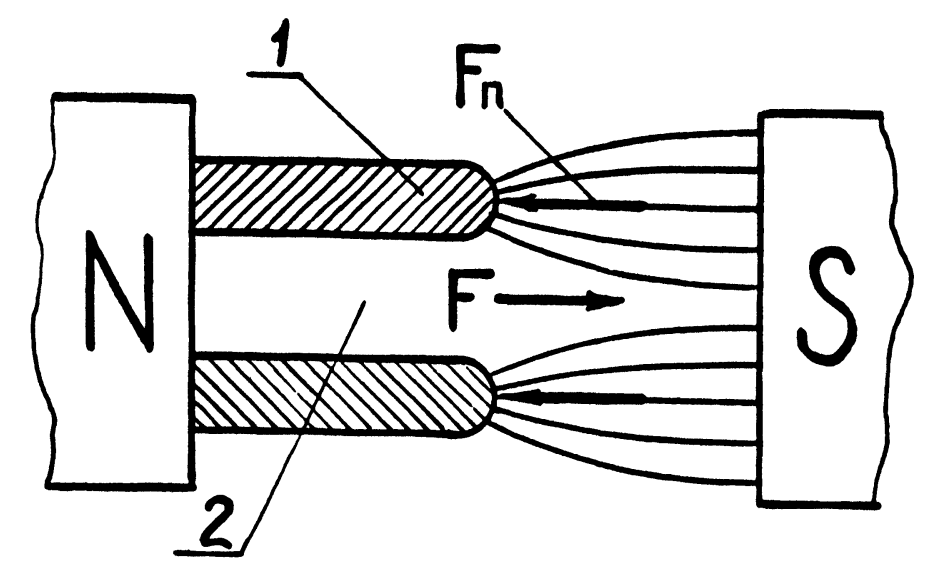

Fig. 1 Schematic diagram of formation of the repulsive magnetic force

Figure 1 illustrates the magnetic principle on which the method is based. There are two ferromagnetic bodies 1 between pole-pieces of the magnetic circuit. These ferromagnetic bodies are arranged with a gap 2 between them. The magnetic flux 
lines. The magnetic flux lines are deflected and concentrated in the front edges of the ferromagnetic bodies, forming regions of attractive magnetic force $F_{\mathrm{n}}$. As a result, the magnetic field intensity is weaker in the region adjacent to the gap between the ferromagnetic bodies than at a distance from the gap. Therefore, the magnetic force $F$ acting along the field is in the direction away from the gap. This force represents the magnetic barrier in the way of the movement of the material to be separated.

A static separator shown in Figure 2 comprises a magnetic circuit 1 and a matrix mounted in the gap between the pole-pieces. The matrix includes an array of elongated ferromagnetic bodies in the form of triangular plates 2 The front edge 3 of each plate is oriented at an angle substantially perpendicular to the direction of the magnetic field, and at an acute angle to the vertical line.

As a result of such orientation, the magnetic force is generated in the space between the front edges of the ferromagnetic plates and is directed outwards from the gaps between these plates. The front edge of each plate is rounded off in its cross-section and has a protective plate 4 of non-magnetic material attached to it. This non-magnetic plate covers the region of attractive magnetic force. The magnetic and non-magnetic plates are attached to each other forming walls of the separation channels 5 .

As a result of such an arrangement of the plates, the magnetic barriers formed by the repulsive magnetic force are located inside separation channel arranged in the way of the material to be separated. The attractive magnetic force is located inside non-magnetic plates and does not have any influence on the separation process. Every separation channel has a divider 6 in its upper part, feeders for material ( 7 ) and water (8) and discharge channel for non-magnetic (9) and magnetic (10) fractions.

A material to be separated in the form of a slurry along the separation channel enters the gaps between the ferromagnetic plates. The magnetic particles (denoted by solid circles) cannot overcome the magnetic barrier and are deflected by the repulsive magnetic force from the gaps between the ferromagnetic plates. They are thus propelled along the front edges of the plates towards the discharge channel for the magnetic fraction. 


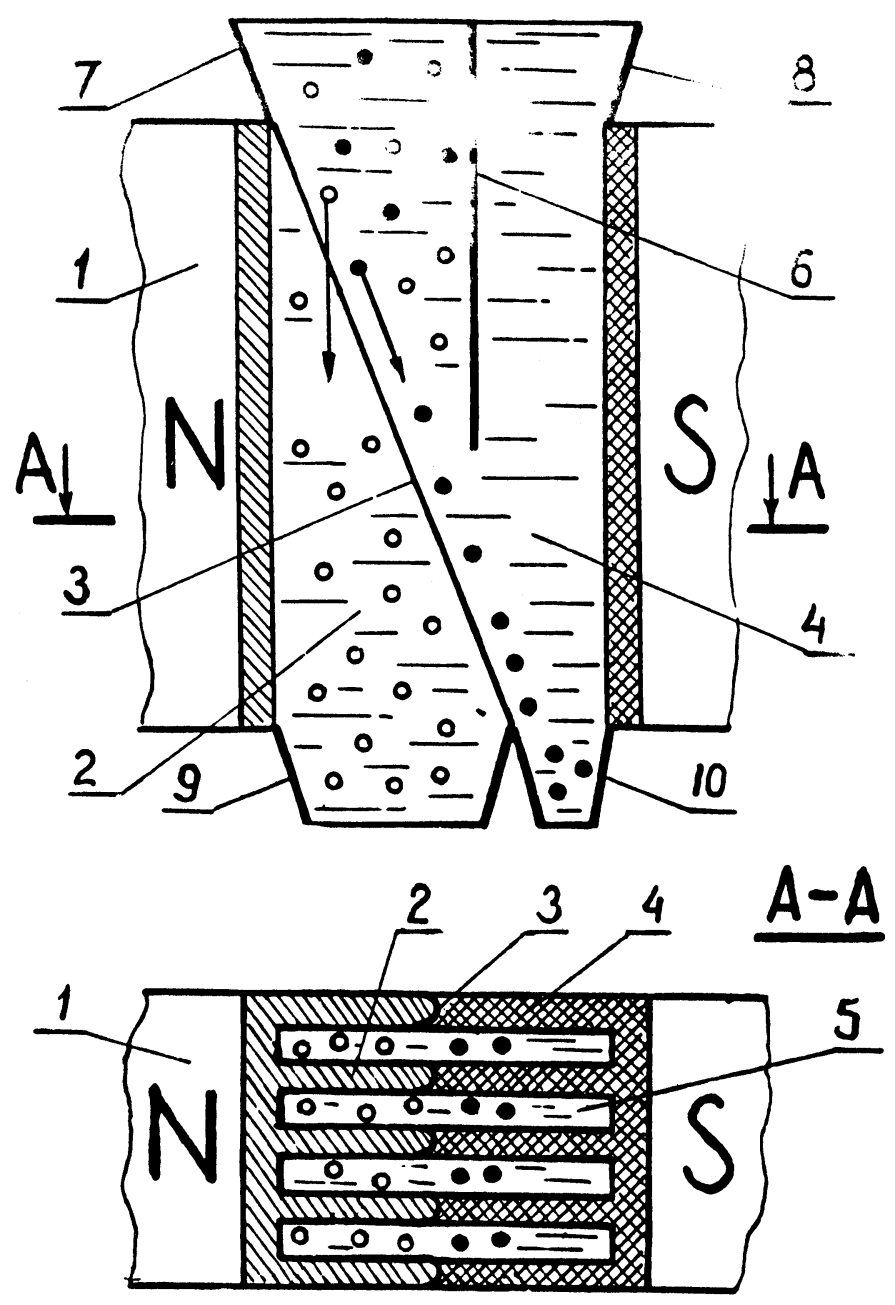

Fig. 2 Schematic diagram of a static matrix separator for continuous separation.1-magnetic circuit, 2-triangular ferromagnetic plates, 3-front edge of the plate, 4-non-magnetic plate, 5-separation channels, 6-divider, 7-material feeder, 8-water feeder, 9-non-magnetic fraction, 10-magnetic fraction

The non-magnetic particles (indicated by open circles) pass through the space between the ferromagnetic plates and report into the discharge trough for non-magnetic fraction. The magnetic particles cannot be attracted to the front 
edges of the ferromagnetic plates as it is prohibited by the non-magnetic plates.

Investigation of this new method of separation was carried out at the Institute of Geotechnical Mechanics of the Ukrainian Academy of Sciences. A laboratory-scale separator was developed for this purpose. Electromagnetic circuit provides a horizontal magnetic field up to 1.3 Tesla. The matrix of the separator consisted of a pair of triangular ferromagnetic plates arranged with $4 \mathrm{~mm}$ clearance between them. The front edges of the plates were inclined at an angle of $4^{\circ}$ to the vertical.

The laboratory separator was used to implement a wet separation process. Manganese ore in the particle size range $-1 \mathrm{~mm}$ was treated. The $\mathrm{Mn}$ concentration in the ore was 16.7 to $17.1 \%$. The results of separation are shown in Table 1. For the purpose of comparison, the ore was also treated using matrices similar to the used in the Jones and 6 ERM-35/315 separators.

Table 1 Comparison of the results of magnetic separation of a manganese ore

\begin{tabular}{|l|c|c|c|}
\hline \multirow{2}{*}{\multicolumn{1}{|c|}{ Parameter }} & \multicolumn{3}{|c|}{ Matrics of the separator } \\
\cline { 2 - 4 } & Jones & 6 ERM-35/315 & $\begin{array}{c}\text { for the continuous } \\
\text { separation }\end{array}$ \\
\hline Clearance between plates, mm & 2 & 4 & 4 \\
Thickness of plates, mm & 4 & 4 & 4 \\
Height of plates, mm & 200 & 200 & 200 \\
Magnetic flux density, T & 1.25 & 1.25 & 0.9 \\
Solid content of a slurry, \% & 20 & 20 & 20 \\
Output of a separator, t/h & 40 & 70 & 100 \\
Content of maganese into & & & 0.7 \\
\multicolumn{1}{|c}{ tails, \% } & 12 & 4.5 & 39.9 \\
concentrats, \% & 37.4 & 38 & 40.8 \\
Yield of concentrate, \% & 20.1 & 36 & 97.5 \\
\hline
\end{tabular}


In accordance with the principle of action of the Jones separator, the slurry passed through narrow $2 \mathrm{~mm}$ gaps between ferromagnetic plates which have vertical ridges and grooves. Movement of the slurry in the matrix of the separator 6 ERM-25/135 was implemented in the form of a film along the walls of the plates with grooves inclined on opposite sides of a wide gap in different directions. The gap was $4 \mathrm{~mm}$ wide. Such large clearances between the plates permit to reduce clogging of the gaps and to increase the throughput of the separator [2].

Throughput of the separator is an expected throughput with power of the magnetic system and area of the matrix similar to that the 6 ERM-35/135 separator. The results shown in Table 1 indicate that the worst results were obtained using the matrix used in the Jones separator while the matrix employed in the 6 ERM-35/135 gave intermediate results.

The best results were obtained using the matrix for continuous separation. Comparing these results with those obtained with the 6 ERM-35/135 matrix, it can be seen that the quality of the concentrate increased by $1.9 \%$, recovery of manganese increased by $14.7 \%$ and throughput of the separator by $30 \%$. The required magnetic flux density decreased by $30 \%$. Considerable technological superiority of the static matrix was achieved when quartz sand, garnet and other materials were treated.

\section{REFERENCES}

[1] A.M. Turkenich, E.S. Lapshin, R.S. Ulubabov and V.I. Dudnik: Industrial Property 6 (Kiev, 1994)

[2] R.S. Ulubabov, A.M. Turkenich: Mining Journal (Moscow), No. 5 (1986)

Alexander M. Turkenich: for biography see Magn. Electr. Sep. 8 (1996), No. 1 


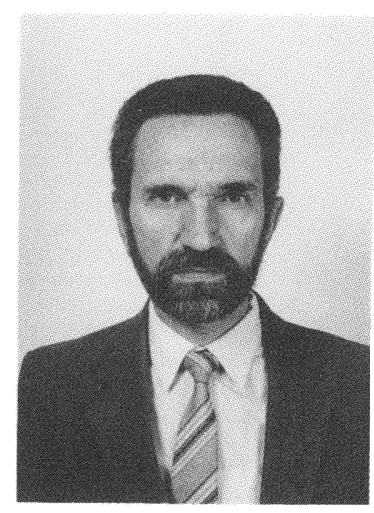

E.S. Lapshin graduated from the Dnepropetrovsk University, Ukraine in 1972. He obtained his $\mathrm{Ph} . \mathrm{D}$. degree in 1990 and his main field of interest is high-gradient magnetic separation.

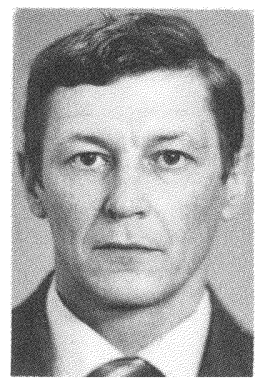

V.I. Dudnik graduated from the Dnepropetrovsk University, Ukraine, in 1972 . He is presently involved in high-gradient magnetic separation.

Keywords: Barrier separation, magnetic separation, magnetic force, repulsive force 